\title{
Simulation of the Load Evolution of an Anchoring System under a Blasting Impulse Load Using FLAC ${ }^{3 D}$
}

\author{
Xigui Zheng, Jinbo Hua, Nong Zhang, Xiaowei Feng, and Lei Zhang \\ School of Mines, Key Laboratory of Deep Coal Resource Mining, Ministry of Education of China, \\ China University of Mining and Technology, Xuzhou, Jiangsu 221116, China
}

Correspondence should be addressed to Nong Zhang; zhangnong@126.com

Received 1 August 2014; Accepted 28 November 2014

Academic Editor: Alicia Gonzalez-Buelga

Copyright (C) 2015 Xigui Zheng et al. This is an open access article distributed under the Creative Commons Attribution License, which permits unrestricted use, distribution, and reproduction in any medium, provided the original work is properly cited.

\begin{abstract}
A limitation in research on bolt anchoring is the unknown relationship between dynamic perturbation and mechanical characteristics. This paper divides dynamic impulse loads into engineering loads and blasting loads and then employs numerical calculation software FLAC ${ }^{3 \mathrm{D}}$ to analyze the stability of an anchoring system perturbed by an impulse load. The evolution of the dynamic response of the axial force/shear stress in the anchoring system is thus obtained. It is revealed that the corners and middle of the anchoring system are strongly affected by the dynamic load, and the dynamic response of shear stress is distinctly stronger than that of the axial force in the anchoring system. Additionally, the perturbation of the impulse load reduces stress in the anchored rock mass and induces repeated tension and loosening of the rods in the anchoring system, thus reducing the stability of the anchoring system. The oscillation amplitude of the axial force in the anchored segment is mitigated far more than that in the free segment, demonstrating that extended/full-length anchoring is extremely stable and surpasses simple anchors with free ends.
\end{abstract}

\section{Introduction}

Prestressed anchoring technology, characterized by its high efficiency and profound economic benefits, has been widely applied and highly developed in various civil engineering applications [1]. This innovative methodology is theoretically based on an implied stress transfer mechanism under tension load and is widely used in collieries. Technicians and researchers worldwide have carried out many experiments and field tests to gain a detailed understanding of the mechanisms and performance of the technology.

The mechanism of mechanical transfer between a bolt/ cable and grouting has been investigated [2-8]. Kang focused on the coupling relationship among the bolt prestress, bolt length, and prestressed field distribution using FLAC ${ }^{3 \mathrm{D}}$ software [9]. Lu et al. investigated the stability of a roadway that was affected by adjacent excavations, ultimately obtaining a relationship between the deformation of the roadway and the anchoring force [10]. Moosavi et al. tested the mechanism of anchor failure [11]. Nevertheless, the aforementioned results mainly focused on static mechanical analysis and ignored blasting effects, rock burst impacts, seismic wave disturbance, and similar impulse loads induced by underground mining. Frequent periodic weighting, driving loads, and coal-mining cyclic loading greatly threaten the stability of the surrounding rock mass in mining. This threat is even more prominent for deep rock masses for which stress concentrations are higher and dynamic disturbances are fiercer $[12,13]$. Anchoring units are thus affected and eventually destroyed by the evolution of the dynamic load. There is thus a scientific need to study the evolution of the anchoring system under different loads rather than conduct simple static mechanical analysis. Gao et al., for instance, investigated the evolution of the stress field of a rock mass, displacement field, and plastic zone under dynamic load disturbance, thus revealing the relevant mechanism that destabilizes or destroys a deep high-stress roadway in a mine [14]. The authors of this paper numerically studied the stress evolution of a bolt subjected to a dynamic load [15-17].

The present paper employs the dynamics module of FLAC $^{3 \mathrm{D}}$ software to numerically simulate the transfer/evolution of stress along a bolt/cable and the mechanism of mutual stress transfer between an anchoring system and rock mass in the case of impulse dynamic load disturbances such as 
TABLE 1: Initial parameters for fitting curves of shear stress.

\begin{tabular}{ccccccc}
\hline \multicolumn{2}{c}{$\begin{array}{c}\text { Shear modulus of a different lithology/GPa } \\
\text { Stable }\end{array}$} & Moderate stable & Unstable & $\begin{array}{c}\text { Shear modulus of } \\
\text { resin/GPa }\end{array}$ & $\begin{array}{c}\text { Radius of } \\
\text { bolts/m }\end{array}$ & $\begin{array}{c}\text { Radius of } \\
\text { contact face/m }\end{array}$ \\
\hline 5.6 & 2.8 & 1.4 & 1.2 & 0.011 & 0.025 & $\begin{array}{c}\text { Elasticity modulus } \\
\text { of cable/GPa }\end{array}$ \\
\hline
\end{tabular}

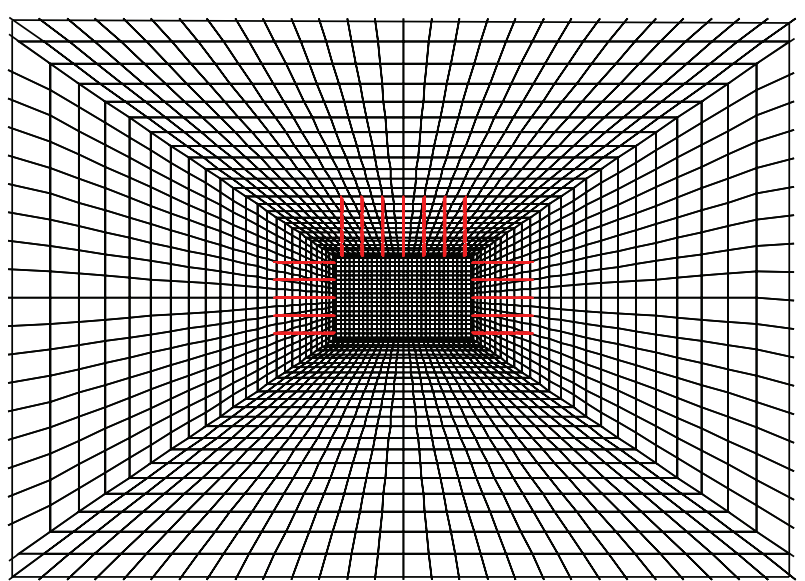

FIgURE 1: Schematic illustration of three-dimensional model.

those generated by blasting, seismic loading, and periodic weighting during mining.

\section{Numerical Model and Schemes}

2.1. Model and Parameter Initiation. A model is established using the Hoek-Brown yield criterion in FLAC ${ }^{3 \mathrm{D}}$. The thickness and depth of the coal seam are specified as 20 and $800 \mathrm{~m}$, respectively. The immediate surroundings and floor comprise moderate stable strata. Relevant parameters are given in Table 1.

The modeled roadway has a rectangular cross-section and is located at the center of the coal seam. The roadway has a width of $5.0 \mathrm{~m}$ and height of $3.0 \mathrm{~m}$. The total model size is $50 \mathrm{~m} \times 15 \mathrm{~m} \times 60 \mathrm{~m}(X \times Y \times Z)$, as schematically illustrated in Figure 1. All free faces except the upper face are fixed. Incipient perpendicular stress is generated by the dead weight of overlying strata. The side pressure coefficient is 1 . The roof of the roadway is supported by 2.8-meter-long bolts with an anchoring length of $1.6 \mathrm{~m}$ and exposed length of $100 \mathrm{~mm}$. A pretension force is applied to the free segments of the bolts. The bolts are spaced $750 \mathrm{~mm}$ in rows that are spaced $1000 \mathrm{~mm}$. The side walls of the roadway are supported by bolts in a scheme similar to that of the bolts supporting the roof but with bolt spacing of $650 \mathrm{~mm}$ and row spacing of $1000 \mathrm{~mm}$. Note that the cell size in the dynamic calculation must be less than $1 / 8$ to $1 / 10$ of the wavelength corresponding to the highest frequency of the input waveform; hence, the maximal cell size is $1 \mathrm{~m}$ for the stress wave employed in this simulation.

The model is first built in a static state in FLAC ${ }^{3 \mathrm{D}}$ and the incipient stress field is equilibrated. The roadway is excavated for a length of $7 \mathrm{~m}$ and the prestressed bolt supporting

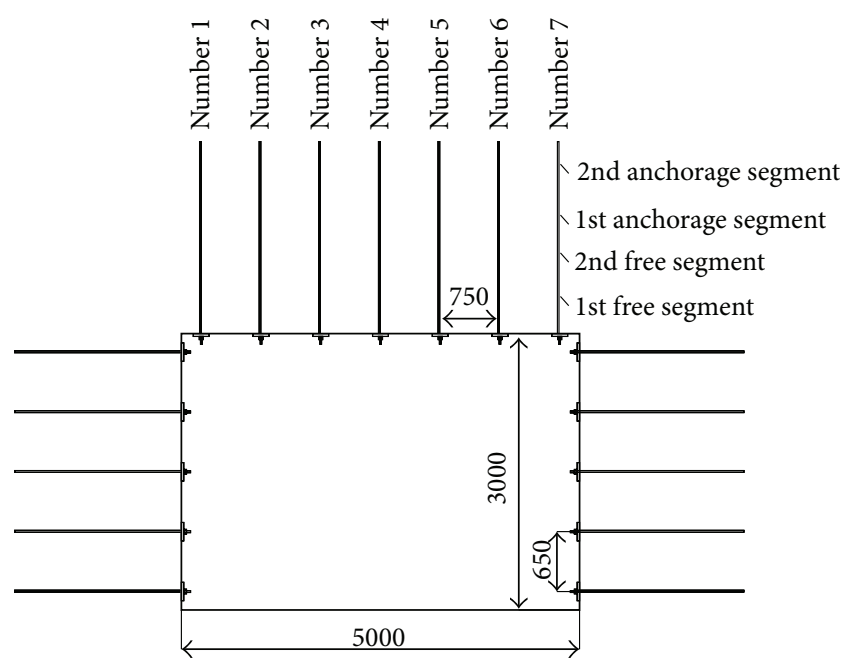

Figure 2: Arrangement and serial numbers of bolts.

scheme described above is then implemented. Each bolt has four units; the first and second free units are both $0.5 \mathrm{~m}$ in length, while the first and second anchoring units are $0.85 \mathrm{~m}$ in length. The anchoring scheme is specified as extended anchoring. A pretension force of $40 \mathrm{kN}$ is applied to bolts and the simulation is allowed to run until equilibrium is reached. The initial fixed boundary is then replaced by a static boundary to begin the dynamic analysis module. An impulse load wave is imposed near the excavated head-on of the roadway so as to simulate the impact of a blasting load on antecedent installed bolts. Because the model and impulse load are symmetrically built and distributed, it is reasonable to analyze half of the roadway for the sake of simplicity. The arrangement and serial numbers of several bolts are presented in Figure 2.

2.2. Optimization of Dynamic Boundary Conditions. To absorb wave energy reflected from the model boundary as much as possible and thus guarantee calculation precision, relevant static boundary conditions and free-field boundary conditions are established.

A power source can be applied to the cells of a model directly to provide the impulse load. There is therefore no need to add an additional free-field boundary, and a static boundary is already capable of effectively reducing the reflection of wave energy. A static boundary and dynamic boundary are shown in Figure 3. All faces except the upper face are fixed to obtain the static solution. Stress of $20 \mathrm{MPa}$ is applied to simulate the dead weight of overlaying strata. In the next procedure of dynamic response model, all these 


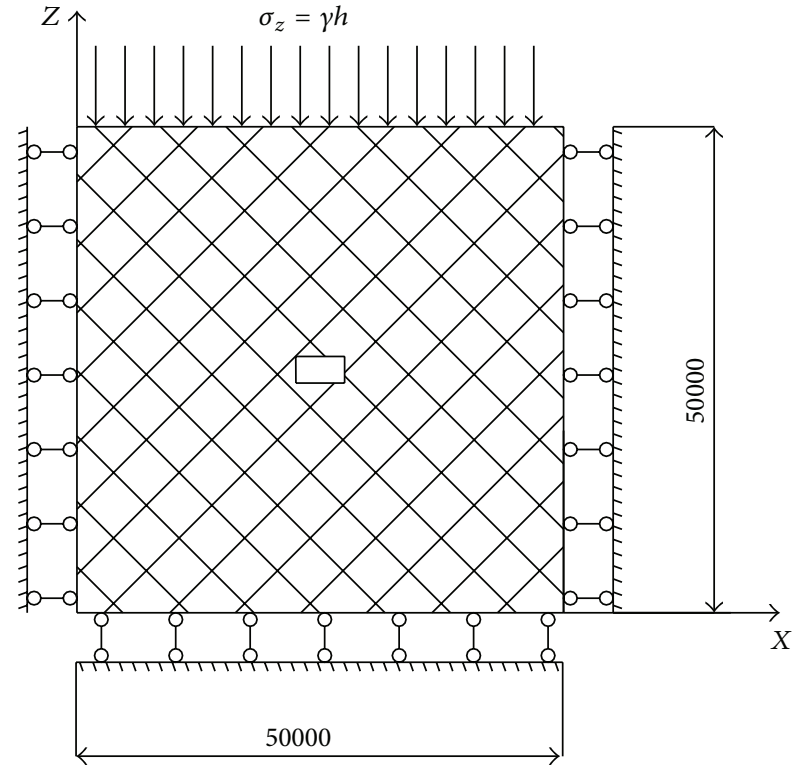

(a)

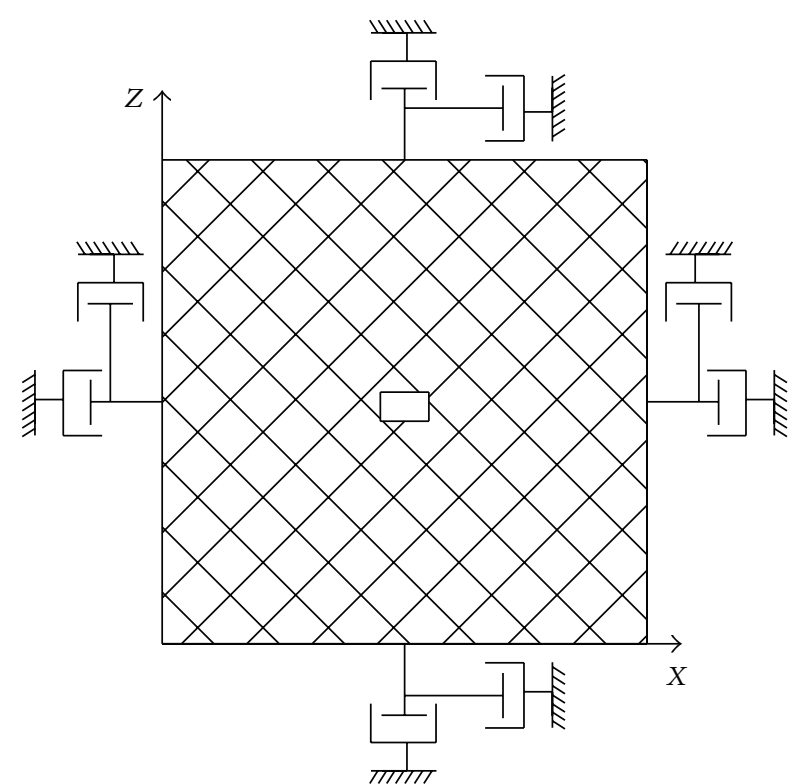

(b)

FIGURE 3: Boundary conditions of the static solution and dynamic solution; (a) boundary conditions of the static model; (b) boundary conditions of the dynamic model.

boundary conditions are removed and viscous boundary conditions are applied to all faces.

2.3. Damping Settings. All propagation media have damping characteristics relating to their inner friction and possible contact surfaces and interfaces. It is thus essential that the maximum extent of reappearing their own undisturbed damping properties and value should be reached during numerical simulation to ensure high reliability. However, the largest problem in dynamic numerical calculation is that damping differs for different values. FLAC ${ }^{3 \mathrm{D}}$ provides Rayleigh damping, local damping, and hysteresis damping as damping schemes.

Rayleigh damping and hysteresis damping are generally applied in rock engineering [18]. The Rayleigh damping scheme involves two parameters: the critical damping ratio and central frequency of vibration. The critical damping ratio can typically be set as $2 \%-5 \%$ or obtained in a laboratory test. However, the application of an elastic-plastic model results in much energy being consumed in plastic flow and thus requires a small critical damping ratio, such as $1 \%$. Local damping is widely applied to structural units in FLAC $^{3 \mathrm{D}} ; 8 \%$ is taken in this analysis. The central frequency is difficult to evaluate but may be obtained for a relatively simple model. The natural vibration frequency should first be determined for the model, and the value is then used as an approximation of the central frequency. This paper employs this methodology to obtain a central frequency of $50 \mathrm{~Hz}$.

2.4. Processing of the Impulse Load. The so-called impulse load is a physical quantity that changes instantaneously and returns to its original value. Such a load is generated in events such as blasting, the sudden breakage of a roof, and seismic activity. Much energy can be generated in the event of an impulse load. Part of the energy is released to adjacent rock mass and triggers the crushing and fracturing of the rock mass. The remainder enters the surrounding rock mass at the speed of splitting propagation, which is characterized by an extremely unstable dynamic wave. This dynamic wave destabilizes the anchoring system.

After a seismic wave has entered the rock mass, the rock not only undergoes compression but also a shear effect. There is thus shear failure within the rock mass; that is, there is a shear wave, or $S$ wave, propagating at lower speed and mainly comprising horizontal fluctuation [19]. This paper focuses on the region close to the impulse source, and the seismic waves, wave speeds, and the like all relate to the primary wave, or $P$ wave. The propagation speed and attenuation of the dynamic wave are strongly related to the mechanical properties of the rock mass. Commonly used equations for the velocities of the $S$ and $P$ waves are

$$
\begin{gathered}
V_{P}=\left[\frac{E(1-\mu)}{\rho(1+\mu)(1-2 \mu)}\right]^{1 / 2}, \\
V_{S}=\left[\frac{E}{2 \rho(1+\mu)}\right]^{1 / 2},
\end{gathered}
$$

where $E$ is the elastic modulus of the propagation medium, $\mu$ is Poisson's ratio, and $\rho$ is the density of the medium.

An oscillogram has a high-frequency component during the postprocessing of impulse data because of the discrete 


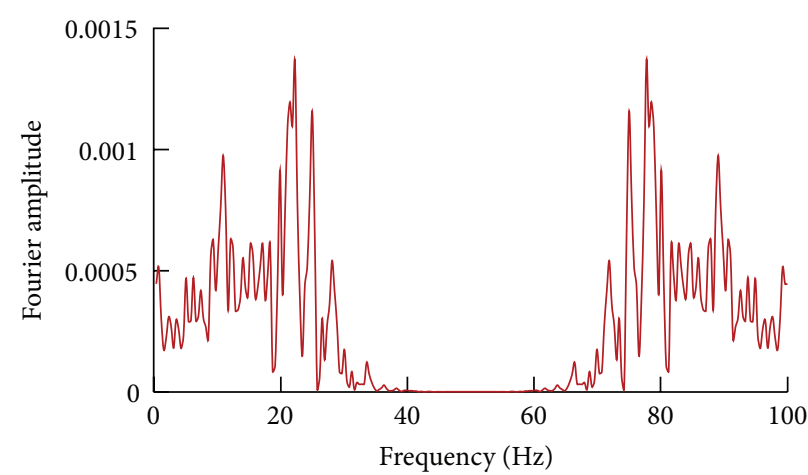

(a) Fourier amplitude

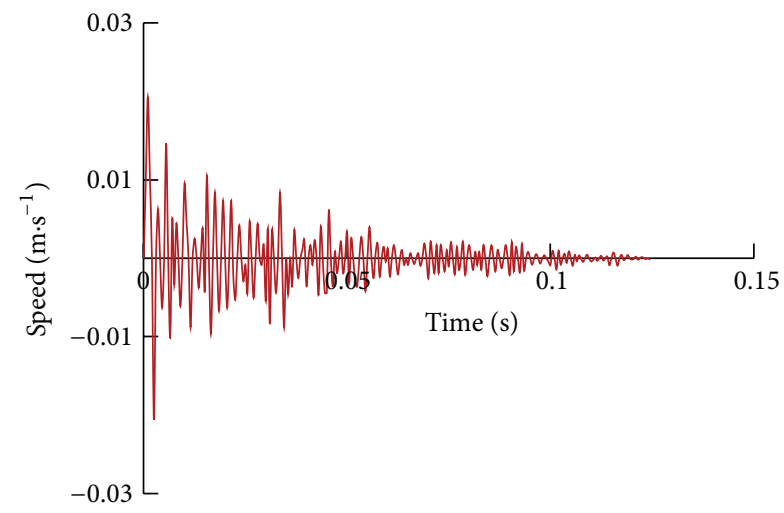

(c) Preprocessing waveform

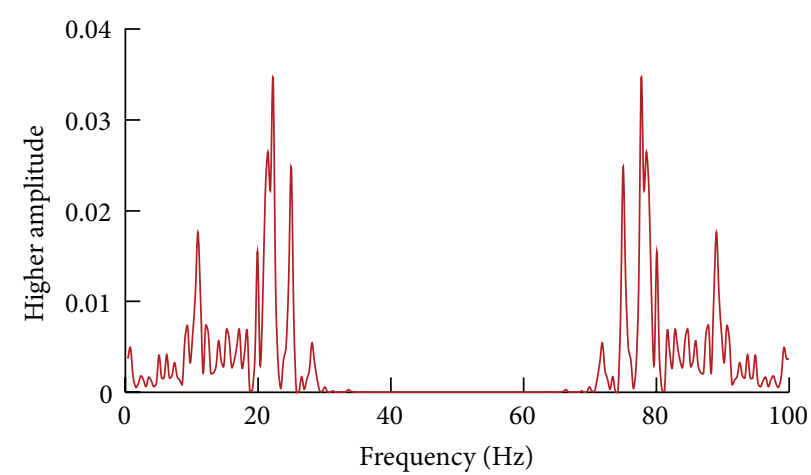

(b) Higher amplitude

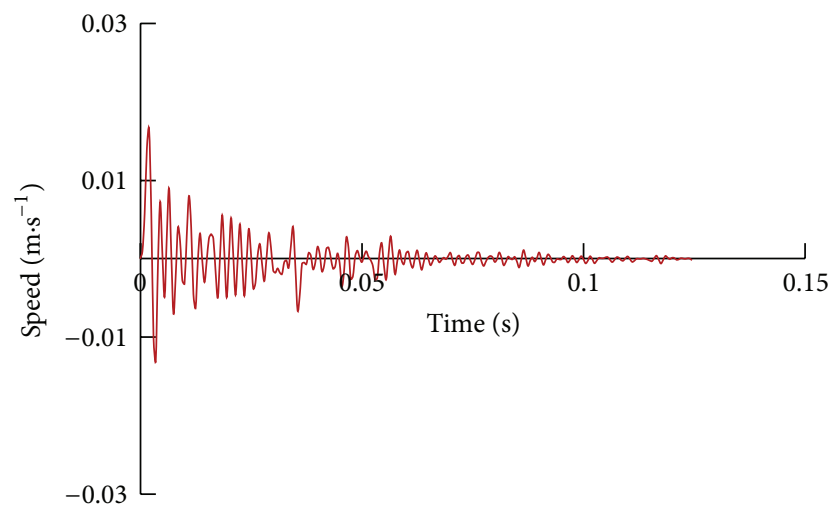

(d) Processed waveform

FIGURE 4: Filtering and baseline correction of the impulse wave source.

measurement of the initial load. The high-frequency component is detrimental to the calculation in the simulation, because as mentioned above the cell size of the grid must be less than $1 / 10$ of the wavelength. The component limits the size of a cell to a very tiny range, and the tiny value is impossible to calculate. It is thus necessary to execute baseline correction and filtering before applying the load so as to filter out the high-frequency component of the wave. It is eventually possible to increase the model cell size and thus reduce the calculation cost and simultaneously diminish potential residual deformation in the dynamic calculation of a plastic constitutive model.

Here Seismic Signal software is adopted for the filtering and baseline correction of the history of the perpendicular load speed [20]. The Butterworth filter is a linear filter that provides the required precision and has a smooth bandpass frequency-response curve and gentle amplitude-frequency curve.

Frequency bands of the primary wave are determined from the Fourier amplitude and higher amplitude in Figure 4 so as to define the chopping frequency. The figure shows that the chopping frequency of the impulse wave selected in this paper is approximately $30 \mathrm{~Hz}$. This frequency is the criterion used in filtering and baseline correction; that is, only waves having frequency lower than $30 \mathrm{~Hz}$ are allowed to pass. The preprocessing data curve and processed data curve are shown in Figure 4.

\section{Discussion and Analysis of Simulation Results}

3.1. Dynamic Response of the Axial Force of the Anchoring System. It is commonly acknowledged that the amplitude of a wave decreases at a decreasing rate during the propagation of the wave in a medium. The attenuation rate is defined as the difference between the amplitude of two adjacent peaks of a wave divided by the amplitude of the first peak.

Figure 5 presents the evolution of the simulated axial force for four bolts placed from the roof center to the roof corner at a distance $0.5 \mathrm{~m}$ from the excavated head-on. As seen in Figure 5(a), the axial force of the free segment of each bolt increases while oscillating under the effect of the incipient impulse wave, and tension and loosening effects occur repeatedly. The rods thus must have high fatigue resistance. The duration of the process is short and the bolts regain their initial stability after approximately $0.05 \mathrm{~s}$; thus, the high-frequency component is mainly concentrated within the first $0.05 \mathrm{~s}$.

Increments in axial forces after applying an impulse load wave at the head-on of the roadway differ among the bolts, with the axial force of bolt number 4 undergoing the largest change because of the central position of bolt number 4 in the roof. Among the first row of bolts in the roof, the axial force of the free segment is the smallest for bolt number 1 , having a value of $50.24 \mathrm{kN}$. The axial force of this bolt surges 


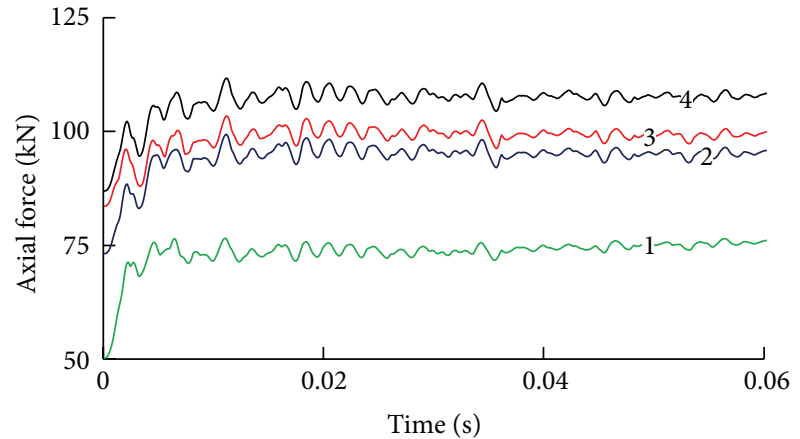

(a)

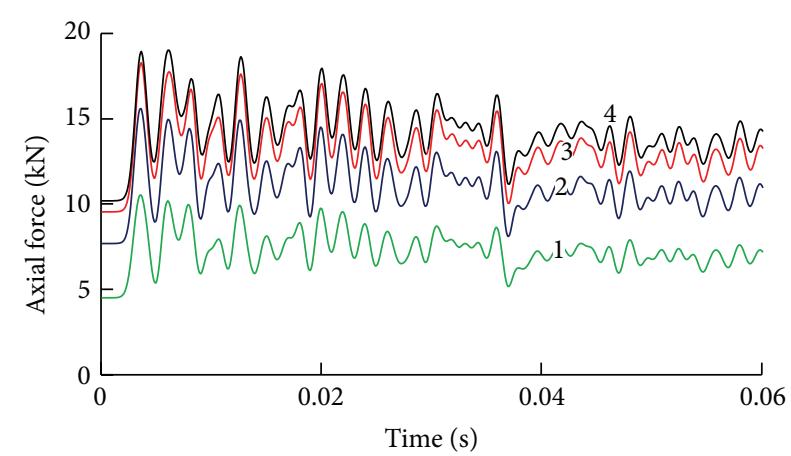

(b)

FIGURE 5: Evolution of axial forces of the first row of bolts in the roof; (a) free segment and (b) anchoring segment.

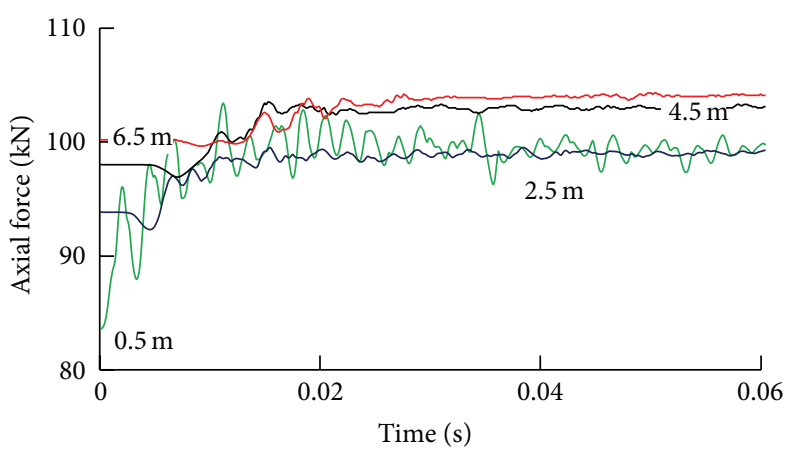

(a)

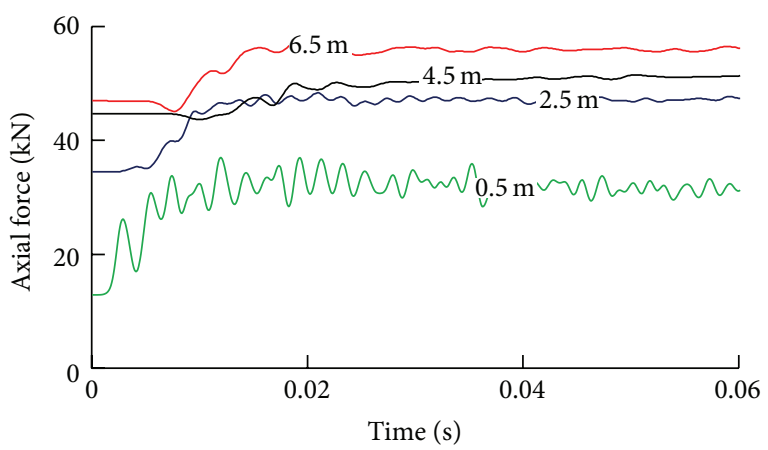

(b)

FIGURE 6: Evolution of the axial force in the simulated anchoring system; (a) evolution of the axial force along the free segment of bolt number 4 ; (b) evolution of the axial force along the anchored segment of bolt number 4.

by $49.56 \%$ to $75.14 \mathrm{kN}$ after the application of the impulse load wave, and the attenuation rate of the first wave cycle is $65.06 \%$. The axial forces of bolts numbers 2, 3, and 4, respectively, increase from initial values of $73.16,83.61$, and $86.93 \mathrm{kN}$ to final values of $94.5,8.61$, and $107 \mathrm{kN}$ (i.e., increases of $29.17 \%$, $17.94 \%$, and $23.08 \%$ ). Apparently, an impulse blasting wave strongly affects the upper corners and central areas, where the anchoring system shows certain instability. The percentage increase in the axial force is greatest for corner bolt number 1 ; this increase is distinctly larger than that for central bolt number 4 . This is explained by (1) the central bolt reaching a stable state earlier than the other bolts and its axial force only slowly increasing with an increase in roof subsidence and (2) bolt number 4 already being highly stressed before the application of the impulse load wave and thus responding little to the dynamic wave.

Figure 5(b) shows that the axial forces of the anchoring segments of the bolts in the first row have an overall increasing trend in response to roadway excavation. The axial force in the anchoring segment of bolt number 1 increases by $52.1 \%$ from 4.51 to $6.86 \mathrm{kN}$ after the application of the impulse load wave, and the attenuation rate in the first wave cycle is $16.49 \%$. Axial forces of bolts numbers 2, 3, and 4, respectively, increase from initial values of $7.69,9.54$, and $10.19 \mathrm{kN}$ to final values of $10.42,12.53$, and $13.30 \mathrm{kN}$ (i.e., increases of $35.50 \%$,
$31.34 \%$, and $30.52 \%$ ). Similar to the case for the free segments, the increment in the axial force is greater for bolt number 1 than for bolt number 4 , and the difference is due to the wave attenuation rate dropping more dramatically than in the case of the free segment. This demonstrates that resin bonds the bolt and rock mass tightly, and the rock mass relieves the shockwave that should initially impact the anchoring system and thus prevents the system from being isolated.

Figure 6(a) presents the evolution of the axial force in the free segment of bolt number 4 for different distances to the entry's head-on (i.e., the central bolts in different rows). It is seen that all of the middlemost bolts in a certain range are affected by the blasting wave and their axial forces have an increasing trend. The increment ratio is a maximum $23.08 \%$ for the bolt $0.5 \mathrm{~m}$ from the head-on, while the ratios for the bolts $2.5,4.5$, and $6.5 \mathrm{~m}$ from the head-on are $5.42 \%, 4.23 \%$, and $3.89 \%$, respectively. The results reveal that a bolt further from the head-on is less affected by the impulse load and has the characteristics of a slower dynamic response, lower oscillation frequency, and lower oscillation amplitude.

Figure 6(b) presents the evolution of the axial force for the anchored segment at different distances to the entry's headon. The characteristics of the evolution under the dynamic are similar to those of the free end shown in Figure 6(a), although the curves in Figure 6(b) have a lower frequency of oscillation 


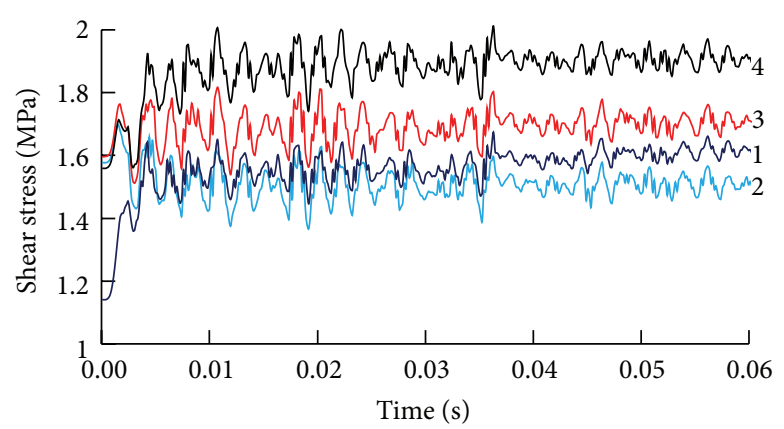

(a)

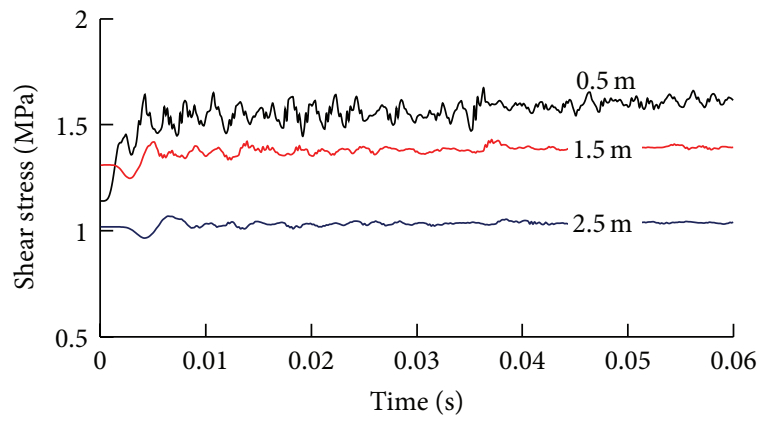

(b)

FIGURE 7: Dynamic response of shear stress along resin in the anchoring system; (a) evolution of shear stress in the anchoring segment of the first row of bolts; (b) evolution of shear stress in the anchoring segment of bolt number 1 for different distances to the head-on.

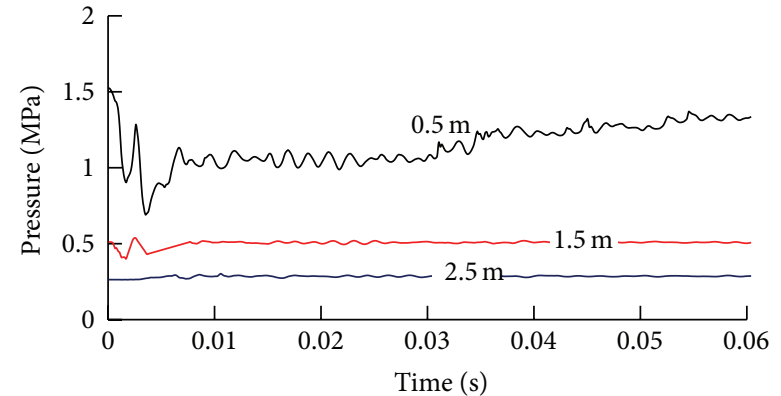

(a)

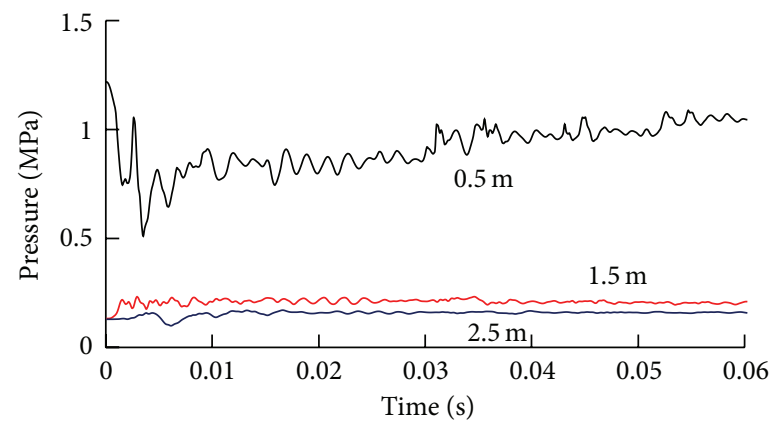

(c)

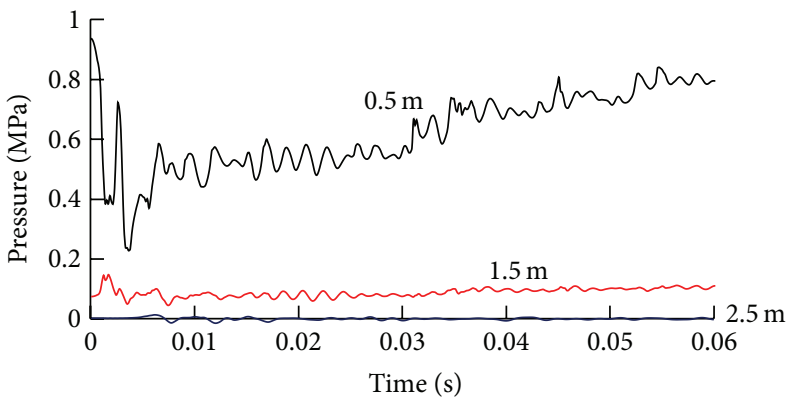

(b)

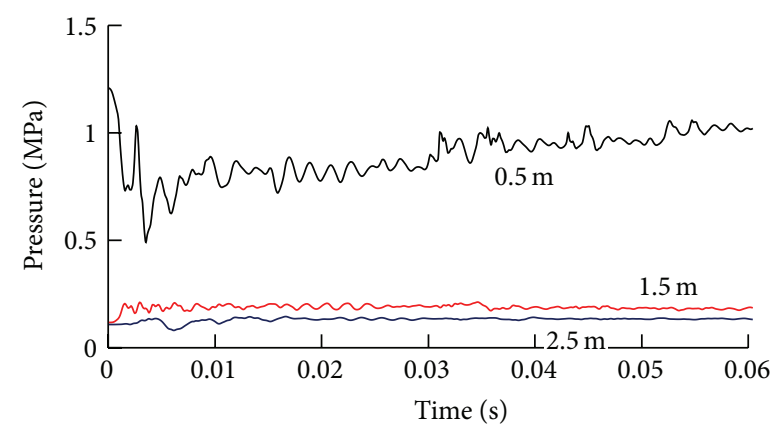

(d)

FIGURE 8: Dynamic response curves for the surrounding rock mass; (a) vertical stress in the roof, (b) vertical stress in the floor, (c) horizontal stress in the right wall, and (d) horizontal stress in the left wall.

and a weaker response, demonstrating that resin mitigates the effect of the blasting impulse load.

3.2. Dynamic Response of Shear Stress along Resin. The dynamic response of shear stress along the resin in a prestressed anchoring system under the effect of a blasting wave is illustrated in Figure 7(a). The shear stress for the anchoring segment of the first row of bolts oscillates dramatically and eventually stabilizes to a certain average value. The shear stress is maximal for central bolt number 4 . Shear stress increment ratios of bolts numbers $1,2,3$, and 4 are $41.37 \%$, $4.50 \%, 6.64 \%$, and $22 \%$, respectively. Accordingly, the bolts most affected are the central and corner bolts, which match the evolution of the axial forces of the bolts. Figure $7(b)$ presents the evolution of the shear stress in the anchoring segment of bolt number 1 for different distances to the headon of the entry (i.e., the corner bolts in different rows). It is seen that the shear stress plummets and the vibration rate reduces as the distance to the head-on increases, revealing that the scope of the effect of excavation blasting on the anchoring system is approximately $1.5 \mathrm{~m}$. The stability of the anchoring system thus requires special attention during blasting so as to guarantee the mechanical properties and active support of the system.

3.3. Dynamic Response Characteristics of Stress in an Anchored Rock Mass. Figure 8 presents dynamic load response curves for different positions in the anchored rock mass. It is 
concluded that the rock pressure at all positions oscillates and attenuates with an increase in the distance to the headon under the effect of a blasting impulse load. The reduction of the pressure amplitude is a maximum at a distance $0.5 \mathrm{~m}$ from the head-on of the entry. The reduction is $14.72 \%$ for the left wall of the roadway, $12.86 \%$ for the roof, $14.70 \%$ for the right wall, and $14.58 \%$ for the floor. Additionally, the decrease in amplitude of the stress in the anchored area plummets and almost reaches zero as the distance from the head-on increases; hence, the scope of the effect in the rock mass is approximately $1.5 \mathrm{~m}$.

\section{Conclusions}

(1) Numerical calculation software FLAC ${ }^{3 D}$ was employed to determine the load stability of an anchoring system under the action of an impulse load wave. The results show that the dynamic response of the axial force conforms to the pertinent waveform of the dynamic impulse load on the whole. The strength of the effects of this impulse perturbation depends on the position within the anchoring system, with the corners and middle being most affected. The dynamic response of shear stress also conforms to the corresponding evolution of the axial force on the whole; however, the oscillations of shear stress are larger and affect both the corners and middle of the anchoring system dramatically.

(2) Anchoring rods are greatly affected by repeated tension and loosening under the perturbation of an impulse load. This means that the rods must be highly resistant to such perturbation. The oscillation amplitude of the anchoring segment is much lower than that of the free segment, revealing that resin acts as an effective buffer that absorbs a certain amount of the impulse load. It is thus beneficial to employ extended or full-length anchoring and thus reduce interference in engineering.

(3) The stress state in an anchored rock mass may decrease under frequent effects, including blasting impulse loads, of head-on excavation. The effects are strongest close to the source of the blasting impulse load. There is eventually stress loss of the initially intact anchored rock mass that reduces the stability of the anchoring system.

\section{Conflict of Interests}

The authors declare that there is no conflict of interests regarding the publication of this paper.

\section{Acknowledgments}

Financial support for this paper was provided by the National Natural Science Foundation of China (51204159) and Fundamental Research Funds for the Central Universities (2011QNB05), and the Qing Lan project is gratefully acknowledged.

\section{References}

[1] W. F. Cofer, D. S. Matthews, and D. I. McLean, "Effects of blast loading on prestressed girder bridges," Shock and Vibration, vol. 19, no. 1, pp. 1-18, 2012.
[2] L. Lutz and P. Gergeley, "Mechanics of band and slip of deformed bars in concrete," Journal of American Concrete Institute, vol. 64, no. 11, pp. 711-721, 1967.

[3] N. W. Hansor, "Influence of surface roughness of pre-stressing strand on band performance," Journal of Pre-stressed Concrete Institute, vol. 14, no. 1, pp. 32-45, 1969.

[4] Y. Goto, "Cracks formed in concrete around deformed tension bars," Journal of American Concrete Intitute, vol. 68, no. 4, pp. 244-251, 1971.

[5] H. X. Jiang, C. L. Du, S. Y. Liu, and K. D. Gao, "Numerical simulation of rock fragmentation under the impact load of water jet," Shock and Vibration, vol. 2014, Article ID 219489, 11 pages, 2014.

[6] X. Y. Wang, J. B. Bai, G. L. Guo, and Y. Yu, “Test and application of hydraulic expansion bolts in a roadway under goaf with ultraclose separation," International Journal of Mining Science and Technology, vol. 24, no. 6, pp. 839-845, 2014.

[7] M. Ghadimi, K. Shahriar, and H. Jalalifar, "Analysis profile of the fully grouted rock bolt in jointed rock using analytical and numerical methods," International Journal of Mining Science and Technology, vol. 24, no. 5, pp. 609-615, 2014.

[8] C. Cao, T. Ren, and C. Chris, "Introducing aggregate into grouting material and its influence on load transfer of the rock bolting system," International Journal of Mining Science and Technology, vol. 24, no. 3, pp. 325-328, 2014.

[9] H. P. Kang, "Development and application of pre-stress anchored bolt supporting technology in coal mine," Coal Mining Technology, vol. 16, no. 3, pp. 25-30, 2011.

[10] S. L. Lu, G. B. Fu, and L. Tang, "Regularity of deformation of rocks around roadway under mining influence and change of rockbolt resistance," Journal of China University of Mining and Technology, vol. 28, no. 3, pp. 201-203, 1999 (Chinese).

[11] M. Moosavi, W. F. Bawden, and A. J. Hyett, "Mechanism of bond failure and load distribution along fully grouted cable-bolts," Transactions of the Institution of Mining and Metallurgy, Section A: Mining Technology, vol. 111, pp. A1-A12, 2002.

[12] M.-Y. Wang, Z.-P. Zhou, and Q.-H. Qian, "Tectonic, deformation and failure problems of deep rock mass," Chinese Journal of Rock Mechanics and Engineering, vol. 25, no. 3, pp. 448-455, 2006 (Chinese).

[13] M.-C. He, H.-P. Xie, S.-P. Peng, and Y.-D. Jiang, "Study on rock mechanics in deep mining engineering," Chinese Journal of Rock Mechanics and Engineering, vol. 24, no. 16, pp. 2803-2813, 2005 (Chinese).

[14] F. Q. Gao, X. F. Gao, and H. P. Kang, "FLAC analysis of mechanical response of surrounding rock mass in deep tunnel," Chinese Journal of Underground Space and Engineering, vol. 5, no. 4, pp. 680-685, 2009 (Chinese).

[15] X.-G. Zheng, L. Zhang, N. Zhang, and N. Liu, "Pre-stressed anchorage function and evolution in the roof of rectangular roadway in deep mine," Journal of China University of Mining and Technology, vol. 42, no. 6, pp. 929-934, 2013 (Chinese).

[16] X. G. Zheng, Evolutionary mechanism of Bolt's(Cable's) lift anchoring force and surrounding rock controlling technology of roadway in coalmine [Ph.D. thesis], China University of Mining and Tecnology, Xuzhou, China, 2013 (Chinese).

[17] L. Zhang, Anchorage load evolution mechanism and control technology under dynamic load in roadway [Master dissertation], China University of Mining and Tecnology, Xuzhou, China, 2014, (Chinese). 
[18] H.-Y. Han and S. Shuang, "Evolution of nonlinear seismic waves in microstructured earth crust," Acta Physica Sinica, vol. 61, no. 5, Article ID 059101, 6 pages, 2012 (Chinese).

[19] G. Fang and B. Zhang, "Lagrangian dynamics and seismic wave align of elastic medium," Acta Physica Sinica, vol. 62, no. 15, Article ID 154502, pp. 1-6, 2013 (Chinese).

[20] R. Zhang, X.-H. Zhao, Y.-J. Hu et al., "A new method of background elimination and baseline correction for the first harmonic," Acta Physica Sinica, vol. 63, no. 7, Article ID 070702, 8 pages, 2014 (Chinese). 

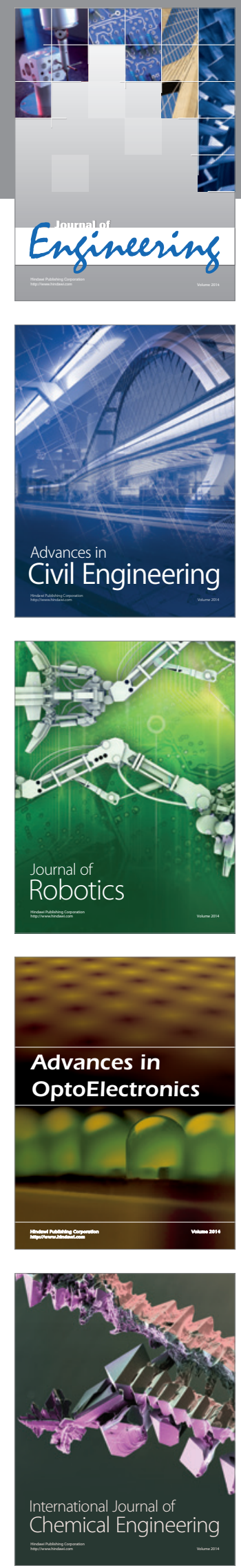

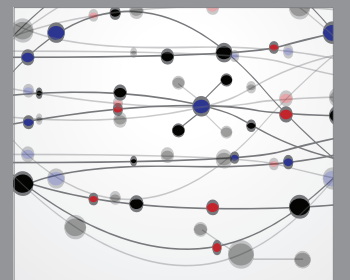

The Scientific World Journal
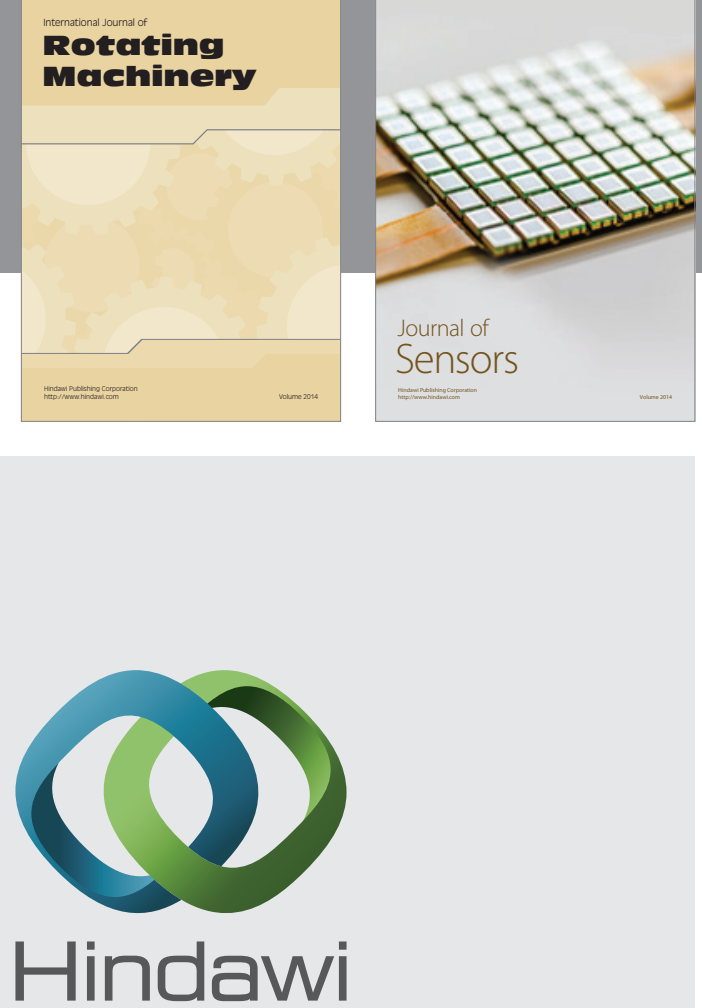

Submit your manuscripts at http://www.hindawi.com
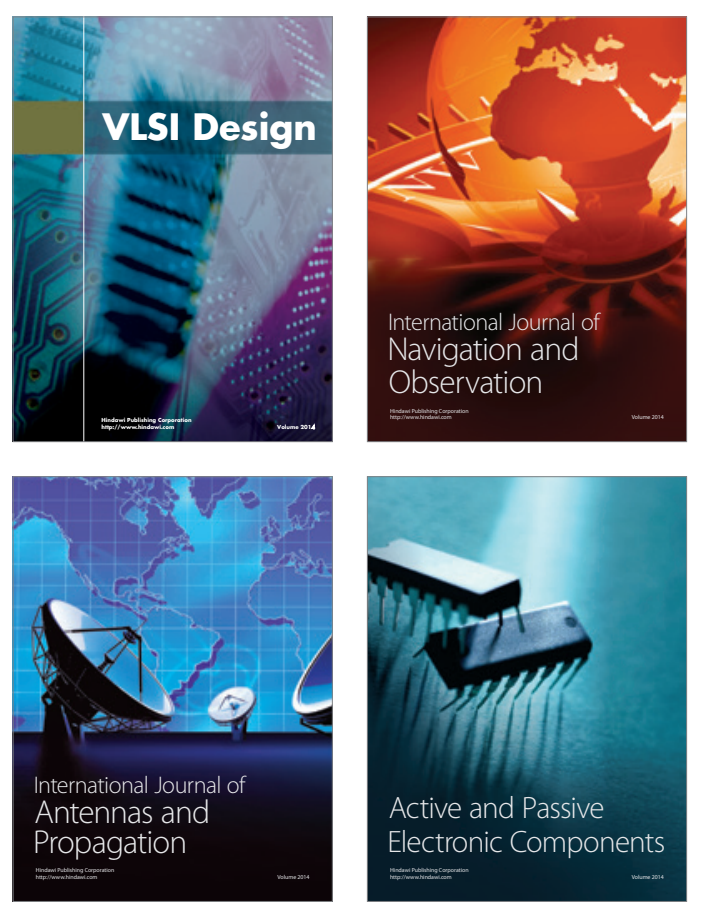
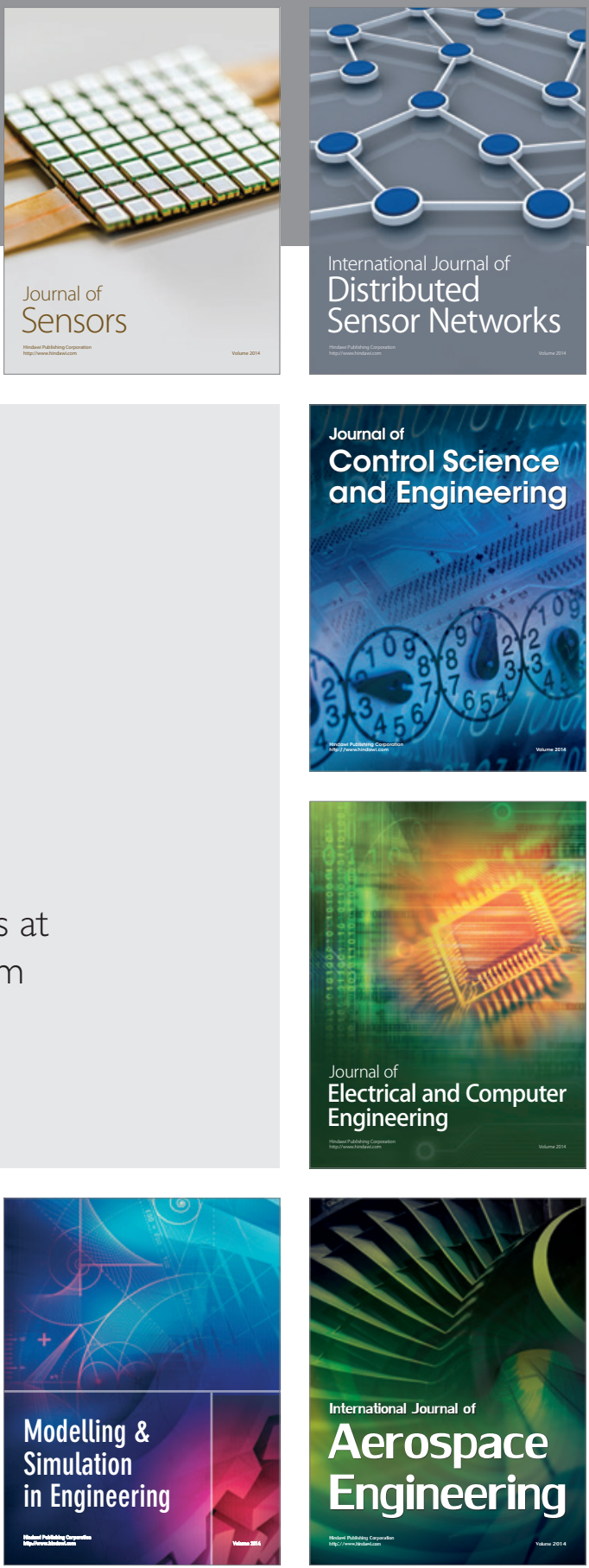

Journal of

Control Science

and Engineering
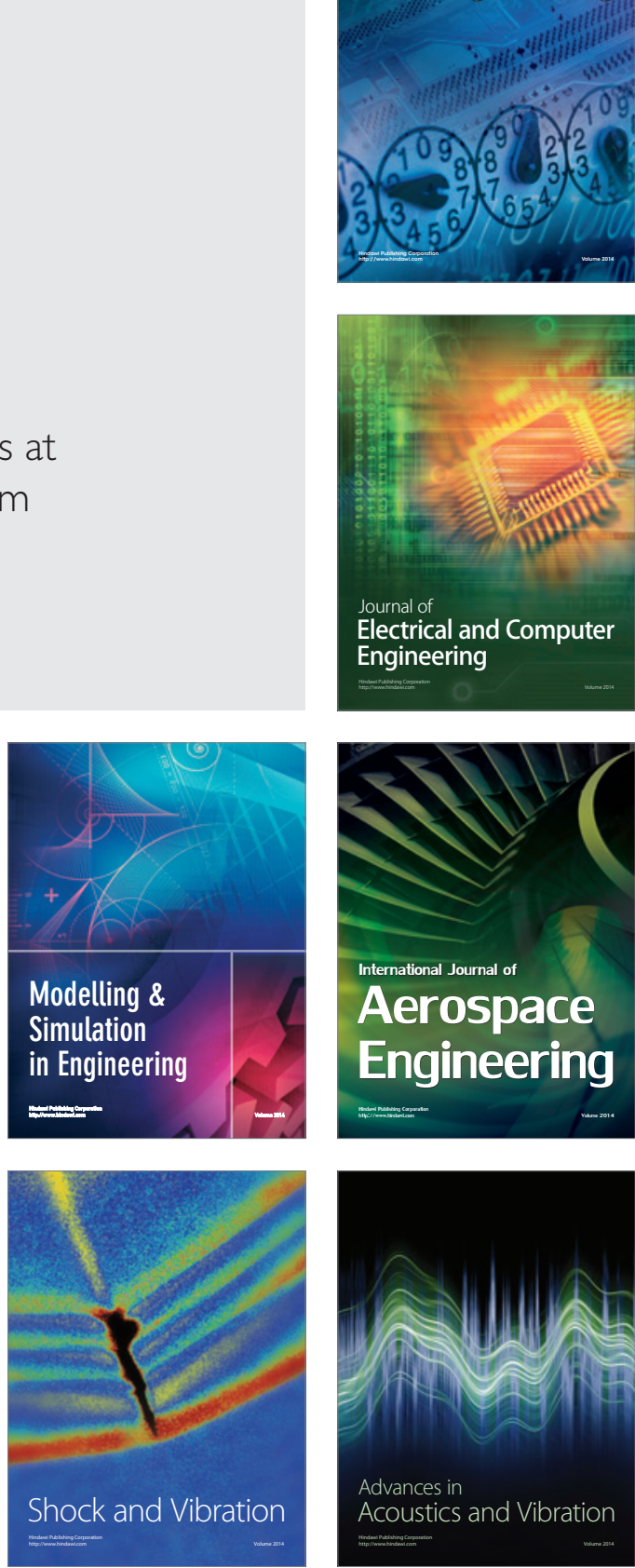